$\begin{array}{ll}\text { Research Square } & \text { Preprints are preliminary reports that have not undergone peer review. } \\ \text { They should not be considered conclusive, used to inform clinical practice, } \\ \text { or referenced by the media as validated information. }\end{array}$

\title{
Surgical Technique and Outcome of Joint Sparing Limb Salvage Surgery by Three-dimensional-printed Prothesis for Children With Osteosarcoma in Distal Femur
}

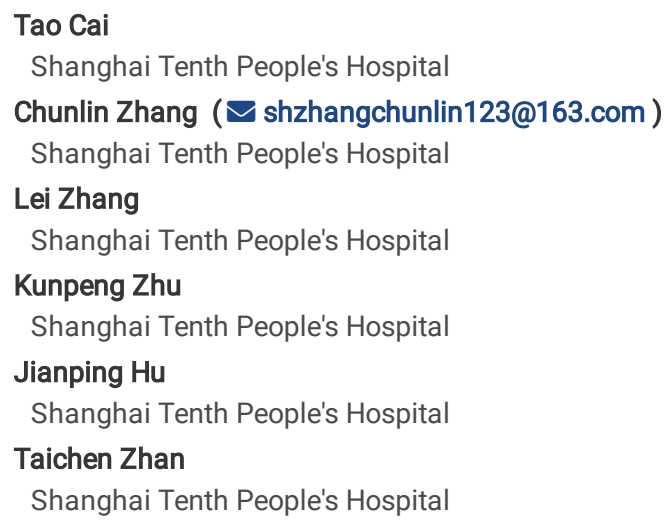




\section{Abstract}

Purpose The purpose of this study was to introduce the surgical technique and functional outcome of joint-sparing limb salvage surgery with metaphyseal osteotomy by dependent design of three-dimensional printed prothesis for children with osteosarcoma in distal femur.

Methods Between 2012 to 2019, we performed twelve joint sparing limb salvage surgeries (JSLSS) with metaphyseal osteotomy for the children of osteosarcoma in the distal femur with our dependent design of 3D-printed guided plate and prothesis. The clinical assessment including resection margin, limb-length discrepancy, orthopaedic complications and functional outcome would be analyzed.

Results The mean operation time for JSLSS was 107.6 minutes and the average blood loss was 194.5ml with the dependent design of 3D-printed prosthesis. With the assistance of guide plate, the mean margin of the osteosarcoma from the epiphyseal plate was $1.6 \mathrm{~cm}$ and the mean limb-length discrepancy was $2.2 \mathrm{~cm}$ with maximum follow-up period of seven years (mean 45.3 months, range 12-84 months). All the patients could bend their knee through $>90^{\circ}$ flexion, achieved similar results for ROMs in 6DOF during gait compared with normal data of Chinese knees, and the mean MSTS score was 94.7 for the patients with JSLSS. One patient had a superficial infection and there was one patient of local recurrence happened during the follow-up with the necrosis rate less than $90 \%$ by preoperative chemotherapy. None aseptic loosening happened in distal femur for the patients in this group with dependent design of 3D-printed prosthesis.

Conclusion Joint-sparing limb salvage surgery by metaphyseal osteotomy with the dependent design of 3D-printed prosthesis and guide plate would be a good choice for the children with osteosarcoma in distal femur.

\section{Introduction}

Distal femur is one of the most common sites for the occurrence of osteosarcoma[1]. With the rapid development of chemotherapy drugs and artificial prosthesis,limb salvage surgery is now routinely performed[2]. In order to reduce the risk of local recurrence, patients with osteosarcoma in distal femur usually accept intra-articular resection with wide surgical margin after preoperative chemotherapy[3]. However, some important structures would be resected at the same time according to the traditional $3 \mathrm{~cm}$ wide margin, such as ligaments, tendons and epiphyseal plate[4]. Even more, hip dissociation sometimes would be conducted if the children were younger than 5 years old, or the tumor involved the whole anterior compartment of the thigh[5]. But, longer survival rates increase the importance of limb and joint salvage for these children.

Nowadays, with the rapid development of 3D-printed technology, joint-sparing limb salvage surgery (JSLSS) could make it possible for the patients to save adjacent joint and the epiphyseal plate affected by osteosarcoma at the same time[6-7]. In present study, surgical excision guide plate and customized artificial prosthesis would be dependent designed by 3D-printed technology. The surgical technique and functional outcome for the JSLSS with dependent design of 3D-printed guide plate and personalized prosthesis with metaphyseal osteotomy would be assessed by our study.

\section{Methods}

\subsection{Patients}

We retrospectively reviewed the bone tumor database of our department between 2012 to 2019. the inclusion criteria were as follows: (1) All the patients were diagnosed as osteosarcoma in distal femur. (2) Epiphyseal plate and epiphyseal line were present in all patients. (3) After preoperativechemotherapy, the osteosarcoma could not invade the epiphyseal plate in all patients. All the patients were provided written informed consent, and the study was approved by the Institutional Review Board of Tongji University (Shanghai, China) and was performed in accordance with the ethical standards prescribed by the Helsinki Declaration.

There were 5 male patients and 7 female patients, with a mean age 12.5 years old ( 9 to 15) at the time of surgery. The base protocol of chemotherapy was given as one cycle courses of intravenous cisplatin, doxorubicin, methotrexate and ifosfamideaccording to our chemotherapy regimen.

\subsection{Dependent design of 3D-printed prosthesis and related surgical procedure}

The 3D-printed guide plate would be designed according to the MRI T1 sequence and gadolinium enhancement analysis. Then according to the CT scan of femur, the resection length and residual metaphyseal-epiphyseal bone segment length would be confirmed for the dependent design of 3D-printed prosthesis. The proximal femoral medullary stalk of the prosthesis is biological fixation type by 3D-printed technology, and lateral auxiliary plateprevents the prosthesis from loosening and rotation. The epiphyseal plate prosthesis would be connected to the intramedullary stalk by an adjustable length connecting rod. The distal epiphyseal plate prosthesis would provide four locking screw holes on the inside and outside of the epiphyseal plate, and the screws could be inserted laterally into the epiphyseal plate. Finally, all the data would be sent to the manufacturer (Shengshi ImplantsLtd, Shanghai, China) along with our measurements for resection and residual bone length, as well as our design concepts and wait 6 weeks to have the implant delivered to hospital (Figure 1).

Before the operation, radiologic examination, including X-ray, CT, MRI and nuclide bone scan would be performed for all the patients to evaluate the relationship between osteosarcoma and epiphyseal plate (Figure 2). After general anesthesia, the anterolateral approach of distal femur would be chosen and osteosarcomawould be separated from the surrounding normal tissue. Then, fix the 3D-printed guide plate with two wires and the distal osteotomies 
through the cutting guide slots would be performed, also the proximal osteotomies would be preformed as usual. After that, the implantation of the 3Dprinted joint-saving prosthesis would be conducted with four locking screws in the epiphyseal plate and two locking screws in the femoral (Figure 3 ).

\subsection{Postoperative evaluation and data analysis}

For the patients with dependent design 3D-printed prosthesis, ankle extension, flexion exercises and muscle toning would be started on day one after the surgery and three weeks later, hip flexion and knee flexion exercises need to be started. Four weeks after the surgery, the patients need to get out of bed and walk with crutches and the patients would be able to walk independently two months later.

During the operations, operation time and blood loss would be recorded exactly. At the final follow-up, the post-operative functional results would be evaluated by the Musculoskeletal Tumour Society (MSTS) scores as well as limb-length discrepancy. Besides, active range of motion (ROM) of the knee in six degrees of freedom (6DOF, angle: flexion-extension, internal-external rotation, adduction-abduction; translation: antero-posterior, proximo-distal, mediolateral) during the gait and the maximum flexion of affected knees were calculated and compared with the data of Chinese normal knees[8].

\section{Results}

There were 5 male patients and 7 female patients, with a mean age 12.5 years old ( 9 to 15) at the time of surgery. For all the twelve patients with osteosarcoma in distal femur, all of them accept the JSLSS with the our dependent design 3D-printed prosthesis, and the mean distance of the osteosarcoma from the epiphyseal plate was $1.6 \mathrm{~cm}$ (Table 1).The mean operation time for the prosthesis group was 107.6 minutes and the average blood loss was $194.5 \mathrm{ml}$. With the maximum follow-up period of seven years (mean 45.3 months, range 12-84 months), the mean limb-length discrepancywas 2.2 $\mathrm{cm}$. All the patients could bend their knee through $>90^{\circ}$ flexion and the mean Musculoskeletal Tumor Society (MSTS) score was 94.7 for the patients with dependent design of 3D-printed prosthesis (Table 2). The results of the patients' knee ROMs in 6DOF during gait showed that the patients with dependent design of 3D-printed prothesis could get the almost normal function compared to the data of normal Chinese knees (Table 3). At the same time, one patients had a superficial infection and there was one patient of local recurrence happened during the follow-up with the necrosis rateless than $90 \%$ by preoperative chemotherapy. Gemcitabine and docetaxel would be used for the patient with another cycle of chemotherapy and the patient is alive with disease until now.

\section{Discussion}

With the advancement of chemotherapy drugs and artificial manufacturing system, joint-sparing limb salvage surgery (JSLSS) has been the standard treatment for children with osteosarcoma in order to maintain normal joint function and protect the epiphyseal plate[6]. Biological reconstruction with vascularized fibular graft[9], treated autologous bone[10-11], allograftcombined fibula allograft[12]and segmentaltransport[13] would be acceptable techniques for this kind of patients. But, these reconstruction techniques would be usually associated with frequent complications, such as graft resorption,nonunion, pathological fractures and insufficient demand of weight-bearing[14]. Three-dimensional-printed segmental prosthesis with the advantages of high shape compatibility and osteointegration capacity of the porous surface has gradually been popular for the JSLSS. Recently, Lu et al reported the good postoperative functional outcome of a patient with 3D-printed prosthesis to reconstruct the defect of the proximal tibia bone tumor defect[15]. Besides, Liu et al reported excellent functional resultsand oncological status for the patients with bone tumors in femur or tibia by 3D-printed intercalary[7]. The 3D-printed prosthesis for the patients in our study were designed by ourselves. According to previous research, aseptic loosening would be the most common complication for the patients with prosthesis replacement in distal femur[16-17]. So, the proximal femoral medullary stalk of the prosthesis and the lateral auxiliary plate would be biological fixation type by 3D-printed technology to promote the bone union and prevent the prosthesis from loosening and rotation. Also, since the main force between the prosthesis and epiphysis would be longitudinal gravity, lateral placement of four locking screws could ensure stability of the implant to a large extent. As a result, all patients in our research had good function without aseptic loosening during the follow-up. Also, compared to other reconstruction method, after the operation for the patients with reconstruction by our 3D-printed prosthesis, the child could carry out the weight-bearing activities and rehabilitation exercises early which would be very important for the function of knee.

The main objective treatment for the surgery of osteosarcoma should be the complete resection of tumor with a safe surgical margin[4]. Luis et al reported that the safe surgical margin should be at least $1 \mathrm{~cm}$ with the accurate evaluation by MRI, which could confirm the epiphyseal extension of osteosarcoma successfully[19-20]. With the assistance of surgical guide (Patient-Specific Instruments), Bellanova et al. reported 4 cases about resection of tumors with histologically negative margins for pediatric tibial bone sarcoma[21]. Based on these findings, in our opinion, if the tumors do not invade the epiphyseal plate according to the evaluation by MRI after the first cycle of chemotherapy, the epiphyseal plate could be preserved completely with the assistance of 3Dprinted guide plate even though the surgical margin would be less than $1 \mathrm{~cm}$. Although, there was one patient of local recurrence in remaining bony epiphysis during the follow-up in our research, we thought it would be strongly associated with the insensitivity of preoperative chemotherapy with necrosis rate less than $90 \%$. Actually, the key factor that would impact the local recurrence rates and overall survival rates would be the tumor sensitivity to chemotherapy when adequate margins would be achieved for all patients as reported[22-23].

For the patients with metaphyseal osteosarcoma, resection of the growth plate could cause limb-length discrepancy(LLD)[12].In 1994, Canadell report the technique of physeal distraction to solve this problem, which permits limb-sparing reconstruction without compromising oncological principles[18]. But, the physeal distraction would be painful and need to take very long time. The expandable prosthesis has been design to avoid limb-length inequality. However, the high rate of complications, including breakage and aseptic loosening of the prosthesis, would have a great influence on the application of this technology. Besides, the length of extension would be limited and the function of the lower extremity could not be as well as expected[15].So, another problem is whether the latitudinal growth would be arrested after epiphyseal-preservation surgery with our dependent design 3D-printed prothesis. The LLD 
would also be happened for the patients with epiphysis preservation for the patients in our study, but the shortened distance would besimilar compared to reconstruction of expandable prosthesis which could be corrected by the assistance of insole and the incidence of complications would be much lower[15,26-28] (Table 4). Besides, Takeuchi et al reviewed 12 patients with osteosarcoma who underwent epiphysis preserving tumor excision and found out that epiphysis transverse growth would not be diminishedafter epiphyseal-preservation surgery in their small series of childhood osteosarcoma around the knee which would be very important to maintain the stability of the knee[24]. Also, according to our research, there was absence of epiphyseal collapse after the surgery and all the patients who underwent JSLSS, achieved similar results for ROMs in 6DOF during gait compared with normal data of Chinese knees.

This is the first study that report the surgical technique and outcome of joint-sparing limb salvage surgery for children with osteosarcoma in distal femur with our dependent designed 3D-printed prothesis. We recognize that our research still have some limitations. First, the sample size of the research would be too small and the the follow-up time would be not long enough for some complications to be detected. Second, because of the study's retrospective natureand lack of a control group, it would be difficult to draw a convincingconclusion. Third, our research only focus on the osteosarcoma in the distal femur for the children, future research about different sites and different types of bone tumors with randomized controlled study need to be conducted.

In conclusion, as long as the tumor does not invade the epiphyseal platefor the patients with osteosarcoma in distal femur, the epiphyseal platecould be preserved completely with acute epiphyseal osteotomy by the assistance of 3D-printed guide plate. Also, joint-sparing limb salvage surgery with our dependent design of 3D-printed prosthesiswould be a good choice for the children with osteosarcoma in distal femur.

\section{Declarations}

\section{Ethical approval:}

All study participants provided written informed consent, the study was approved by the Institutional Review Board of the Tongji University (Shanghai, China) and was performed in accordance with the ethical standards prescribed by the Helsinki Declaration.

\section{Consent for publication:}

We obtained permission from participants to publish the data in theJournal of Orthopaedic Surgery and Research. This manuscript has not been published or presented elsewhere in part or in entirety, and is not under consideration by another journal. All study participants provided informed consent, and the study design was approved by the appropriate ethics review boards. All the authors have approved the manuscript and agree with submission to your esteemed journal.

Competing interests:The authors declare that they have no conflict of interest.

Availability of data and materials: Supporting data is available with the corresponding author on request.

Funding: This project was supported by 1,2, Grant from the National Natural Science Foundation of China (No. 81572630, No.81872174) and 3, Shanghai science and technology talents program(No.19XD1402900). 4, Fundamental Research Funds for the Central Universities, grant \# 22120180385

Acknowledgements: None

\section{References}

1.Ayerza M A, Farfalli G L, Aponte-Tinao L, et al (2010) Does increased rate of limb-sparing surgery affect survival in osteosarcoma?[J]. Clinical Orthopaedics and Related Research®468(11): 2854-2859.

2.Li X, Zhang Y, Wan S, et al. (2016) A comparative study between limb-salvage and amputation for treating osteosarcoma[J]. Journal of bone oncology5(1): 15-21.

3.Yao W, Cai Q, Wang J, et al. (2017) Treatment of osteosarcoma around the knee in skeletally immature patients[J]. Oncology letters14(5): 5241-5248.

4.Chen WM, Wu PK, Chen CF, et al (2012) High-grade osteosarcoma treated with hemicortical resection and biological reconstruction. J Surg Oncol 105:825-829.

5. Benedetti M G, Okita Y, Recubini E, et al. (2016) How much clinical and functional impairment do children treated with knee rotationplasty experience in adulthood?[J]. Clinical Orthopaedics and Related Research® 474(4): 995-1004.

6. Shehadeh A M, Isleem U, Abdelal S, et al. (2019) Surgical Technique and Outcome of Custom Joint-Sparing Endoprosthesis as a Reconstructive Modality in Juxta-Articular Bone Sarcoma[J]. Journal of Oncology, 2019:13.

7. Liu W, Shao Z,RaiS,etal.(2020)Three-dimensional-printed intercalary prosthesis for the reconstruction of large bone defect after joint-preserving tumor resection[J]. Journal of Surgical Oncology, 1-8. 
8. Zhang, Y. et al. Motion analysis of Chinese normal knees during gait based on a novel portable system[J]. Gait \& Posture, 2015, 41(3):763-768.

9. Kiss S, Terebessy T, Szöke G, et al. (2013) Epiphysis preserving resection of malignant proximal tibial tumours[J]. International orthopaedics37(1): 99104.

10. Jeon DG, Kim MS, Cho WH, et al (2007) Pasteurized autograft for intercalary reconstruction: an alternative to allograft. Clin Orthop Relat Res 456:203210

11. Kim JD, Lee GW, Chung SH (2011) A reconstruction with extra- corporeal irradiated autograft in osteosarcoma around the knee. J Surg Oncol 104(2):187-191

12. Houdek M T, Wagner E R, Stans A A, et al.(2016) What is the outcome of allograft and intramedullary free fibula (Capanna Technique) in pediatric and adolescent patients with bone tumors?[J]. Clinical Orthopaedics and Related Research®, 474(3): 660-668.

13.Accadbled F, Thévenin Lemoine C, Poinsot E, et al. (2019) Bone reconstruction after malignant tumour resection using a motorized lengthening intramedullary nail in adolescents: preliminary results[J]. Journal of children's orthopaedics13(3): 324-329.

14. Takeuchi A, Yamamoto N, Hayashi K, et al.(2019) Joint-preservation surgery for pediatric osteosarcoma of the knee joint[J]. Cancer and Metastasis Reviews, 1-14.

15. Lu M, Li Y, Luo Y, et al. (2018)Uncemented three-dimensional-printed prosthetic reconstruction for massive bone defects of the proximal tibia[J]. World journal of surgical oncology16(1): 47.

16.Cipriano C A, Gruzinova I S, Frank R M, et al. (2015) Frequent complications and severe bone loss associated with the repiphysis expandable distal femoral prosthesis[J]. Clinical Orthopaedics and Related Research®473(3): 831-838.

17. Zhang C, Hu J, Zhu K, et al. (2018)Survival, complications and functional outcomes of cemented megaprostheses for high-grade osteosarcoma around the knee[J]. International orthopaedics42(4): 927-938.

18. Can adell J, Forriol F, Cara JA. (1994)Removal of metaphyseal bone tumours with preservation of the epiphysis: physeal distraction before excision. J Bone Joint Surg Br. 76:127-132.

19. Hoffer F A, Nikanorov A Y, Reddick W E, et al.(2000) Accuracy of MR imaging for detecting epiphyseal extension of osteosarcoma[J]. Pediatric radiology30(5): 289-298.

20. Aponte-Tinao L, Ayerza M A, Muscolo D L, et al.(2015) Survival, recurrence, and function after epiphyseal preservation and allograft reconstruction in osteosarcoma of the knee[J]. Clinical Orthopaedics and Related Research®,473(5): 1789-1796.

21. Bellanova L, Paul L, Docquier P L. (2013) Surgical guides (patient-specific instruments) for pediatric tibial bone sarcoma resection and allograft reconstruction[J]. Sarcoma, 2013:7.

22. Jeon DG, Song WS, Kong CB, et al.(2013) Role of surgical margin on local recurrence in high risk extremity osteosarcoma: a case-controlled study. Clin Orthop Surg. 5:216-224.

23.Ferrari S, Ruggieri P, Cefalo G, et al. (2012) Neoadjuvant che- motherapy with methotrexate, cisplatin, and doxorubicin with or without ifosfamide in nonmetastatic osteosarcoma of the extremity: an Italian sarcoma group trial ISG/OS-1. J Clin Oncol. 30:2112-2118.

24.Takeuchi A, Yamamoto N, Hayashi K, et al. (2018) Growth of epiphysis after epiphyseal-preservation surgery for childhood osteosarcoma around the knee joint[J]. BMC musculoskeletal disorders, 19(1): 185.

25.Campanacci L , Manfrini M , Colangeli M , et al. (2010) Long-term Results in Children With Massive Bone Osteoarticular Allografts of the Knee for Highgrade Osteosarcoma[J]. Journal of Pediatric Orthopaedics30(8):919-927.

26.Torner F, Segur J M, Ullot R, et al. Non-invasive expandable prosthesis in musculoskeletal oncology paediatric patients for the distal and proximal femur. First results[J]. International orthopaedics, 2016, 40(8): 1683-1688.

27.Staals E L, Colangeli M, Ali N, et al. Are complications associated with the Repiphysis ${ }^{\circledR}$ expandable distal femoral prosthesis acceptable for its continued use?[J]. Clinical Orthopaedics and Related Research ${ }^{\circledR}, 2015,473(9)$ : 3003-3013.

28.Ruggieri P, Mavrogenis A F, Pala E, et al. Outcome of expandable prostheses in children[J]. Journal of Pediatric Orthopaedics, 2013 , $33(3)$ : $244-253$.

\section{Tables}

Table 1 Demographic data of the patients 


\begin{tabular}{|llllll|}
\hline Patient & $\begin{array}{l}\text { Age } \\
\text { (Year) }\end{array}$ & Sex & ES & $\begin{array}{l}\text { Follow-up } \\
\text { (Month) }\end{array}$ & $\begin{array}{l}\text { OS-EP } \\
\text { Margin }\end{array}$ \\
\hline 1 & 9 & M & IIB & 84 & 0 \\
\hline 2 & 11 & M & IIB & 39 & 1.4 \\
\hline 4 & 12 & F & IIB & 54 & 3.2 \\
\hline 5 & 14 & F & IIB & 19 & 2.6 \\
\hline 6 & 10 & M & IIB & 57 & 1.0 \\
\hline 7 & 15 & F & IIB & 43 & 2.1 \\
\hline 8 & 13 & F & IIB & 65 & 1.6 \\
\hline 9 & 14 & F & IIB & 36 & 0.8 \\
\hline 10 & 13 & F & IIB & 46 & 1.5 \\
\hline 11 & 11 & M & IIB & 37 & 1.3 \\
\hline 12 & 15 & F & IIB & 12 & 1.5 \\
\hline Mean & 12.5 & - & - & 45.3 & 1.6 \\
\hline
\end{tabular}

Abbreviations ES, Enneking stage; OS, osteosarcoma; EP, epiphyseal plate.

Table 2 Operation related data and outcome for the patients with 3D-printed prosthesis.

\begin{tabular}{|ll|}
\hline Variables & Value \\
\hline Operation time, mean (SD), min & $107.6(27.9)$ \\
\hline Blood loss, mean (SD), ml & $194.5(13.5)$ \\
\hline Average shortening length of femur, mean (mm) & 22 \\
\hline MSTS scores, mean & 94.7 \\
\hline Complications (\%) & Infection (8.3\%) \\
& Recurrence (8.3\%) \\
\hline Survival Statue: DFS/AWD & $11 / 1$ \\
\hline
\end{tabular}

Abbreviations DFS, disease free survive; AWD, alive with disease ;MSTS,Musculoskeletal Tumor Society score.

Table 3 The results of the patients' knee ROMs in 6DOF during gait (mean \pm standard deviation).

\begin{tabular}{|lllllll|}
\hline Subjects & $\mathrm{F} / \mathrm{E}\left({ }^{\circ}\right)$ & $\mathrm{IR} / \mathrm{ER}\left({ }^{\circ}\right)$ & $\mathrm{VR} / \mathrm{VL}\left({ }^{\circ}\right)$ & $\mathrm{A} / \mathrm{P}(\mathrm{mm})$ & $\mathrm{P} / \mathrm{D}(\mathrm{mm})$ & $\mathrm{ML}(\mathrm{mm})$ \\
\hline Current Study & $51.9 \pm 5.4$ & $9.6 \pm 1.3$ & $7.1 \pm 1.9$ & $11.3 \pm 0.6$ & $12.5 \pm 3.2$ & $8.7 \pm 2.4$ \\
\hline Normative data[8] & 57.4 & 10.9 & 9.3 & 12.8 & 15 & 9.4 \\
\hline
\end{tabular}

AbbreviationsF/E, flexion/ extension; IR/ER, internal/external femoral rotation; VR/VL, varus/valgus; A/P, anterior/posterior femoral translation; P/D, proximal/distal femoral translation ML, medial/lateral femoral translation.

Table 4 Outcome of the JSLSS for the children with different reconstruction methods. 


\begin{tabular}{|c|c|c|c|c|c|c|c|c|c|}
\hline Study & $\begin{array}{l}\text { Sample } \\
\text { Size }\end{array}$ & $\begin{array}{l}\text { Mean } \\
\text { Age }\end{array}$ & Hitology & $\begin{array}{l}\text { Construction } \\
\text { Method }\end{array}$ & $\begin{array}{l}\text { Epiphysis } \\
\text { Preservation }\end{array}$ & $\begin{array}{l}\text { Follow- } \\
\text { up } \\
\text { (months) }\end{array}$ & Complications & $\begin{array}{l}\text { LLD } \\
\text { (Range) }\end{array}$ & $\begin{array}{l}\text { Local } \\
\text { Recurrence(\%) }\end{array}$ \\
\hline $\begin{array}{l}\text { Cara } \\
\text { A[15] } \\
(2014)\end{array}$ & 10 & 10.1 & $\mathrm{OS}(10)$ & $\begin{array}{l}\text { Cemented } \\
\text { Repiphysis } \\
\text { Expandable } \\
\text { Prosthesis }\end{array}$ & NO & 72 & $\begin{array}{l}\text { Fracture (4), Infection(1), } \\
\text { Aseptic loosening (10) }\end{array}$ & $>20 \mathrm{~mm}$ & $20 \%$ \\
\hline $\begin{array}{l}\text { Torner F } \\
{[26]} \\
(2016)\end{array}$ & 6 & 9.5 & OS(6) & $\begin{array}{l}\text { Non- } \\
\text { invasive } \\
\text { Expandable } \\
\text { Prosthesis }\end{array}$ & No & 66.8 & $\begin{array}{l}\text { Deep infection(1), } \\
\text { Metastasis(3) }\end{array}$ & $46.6 \mathrm{~mm}$ & - \\
\hline $\begin{array}{l}\text { Staals E } \\
\mathrm{L}[27] \\
(2015)\end{array}$ & 15 & 8.4 & $\begin{array}{l}\text { Malignant } \\
\text { bone } \\
\text { tumors(15) }\end{array}$ & $\begin{array}{l}\text { Expandable } \\
\text { Prosthesis }\end{array}$ & No & 104 & $\begin{array}{l}\text { Breakage(8),Loosening(1), } \\
\text { Infection(1) }\end{array}$ & $22.55 \mathrm{~mm}$ & $13.3 \%$ \\
\hline $\begin{array}{l}\text { Ruggieri } \\
\text { P [28] } \\
(2013)\end{array}$ & 32 & 8.9 & Sarcomas(32) & $\begin{array}{l}\text { Expandable } \\
\text { Prosthesis }\end{array}$ & No & 48 & $\begin{array}{l}\text { Breakage(2),Loosening(6), } \\
\text { Infection(4) }\end{array}$ & $15-30 \mathrm{~mm}$ & $9.4 \%$ \\
\hline $\begin{array}{l}\text { Present } \\
\text { Study }\end{array}$ & 12 & 12.5 & OS(12) & $\begin{array}{l}\text { 3D-Printed } \\
\text { Prosthesis }\end{array}$ & Yes & 45.8 & Infection(1) & $22 \mathrm{~mm}$ & $8.3 \%$ \\
\hline
\end{tabular}

Abbreviations: OS, osteosarcoma; Ewing, Ewing sarcoma; FVFG, free vascularized fibular graft; LLD, limb-length discrepancy.

\section{Figures}
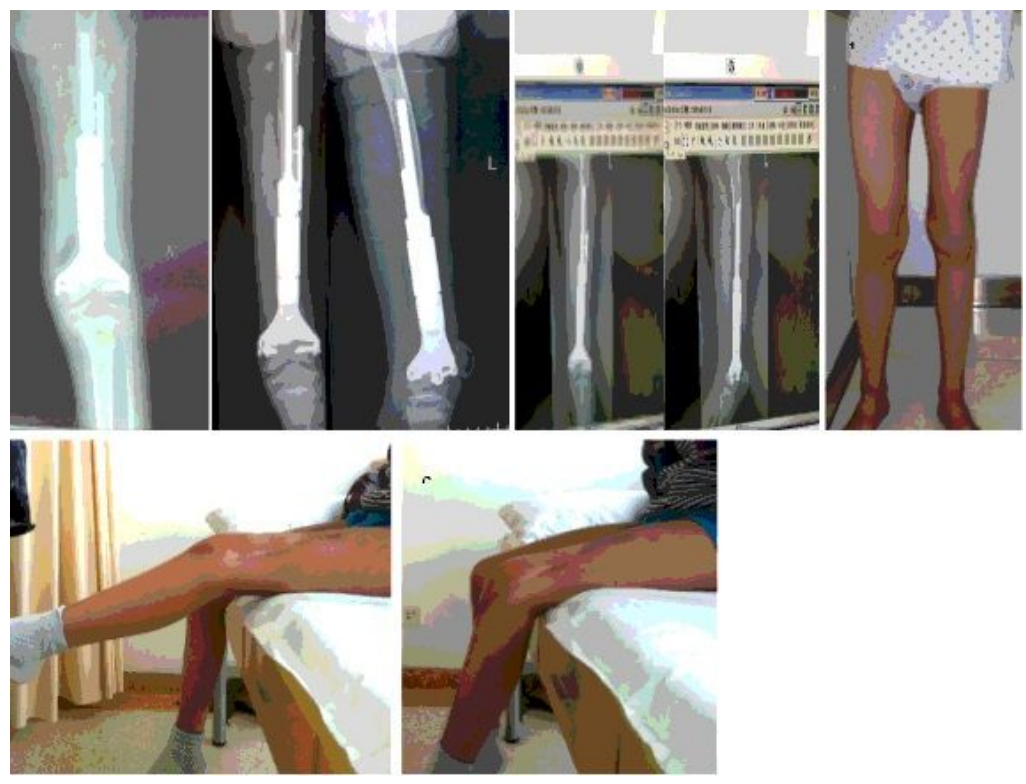

Figure 1

Dependent design of 3D-printed guide plate and prosthesis. (a-e) Schematic diagrams of the design for 3D-printed guide plate and prosthesis. (f-g) Actual view of the 3D-printed prosthesis.
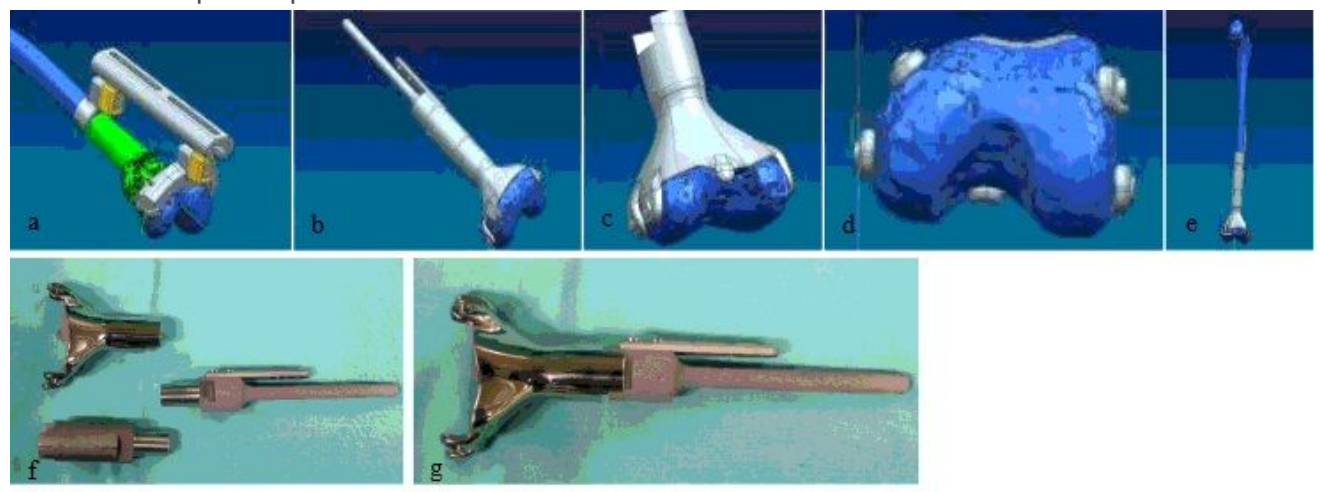


\section{Figure 2}

The radiologic examination for a nine-year-old male child with osteosarcoma in distal femur before operation. (a) X-ray; (b) CT; (c) MRI; (d) Nuclide Bone Scan

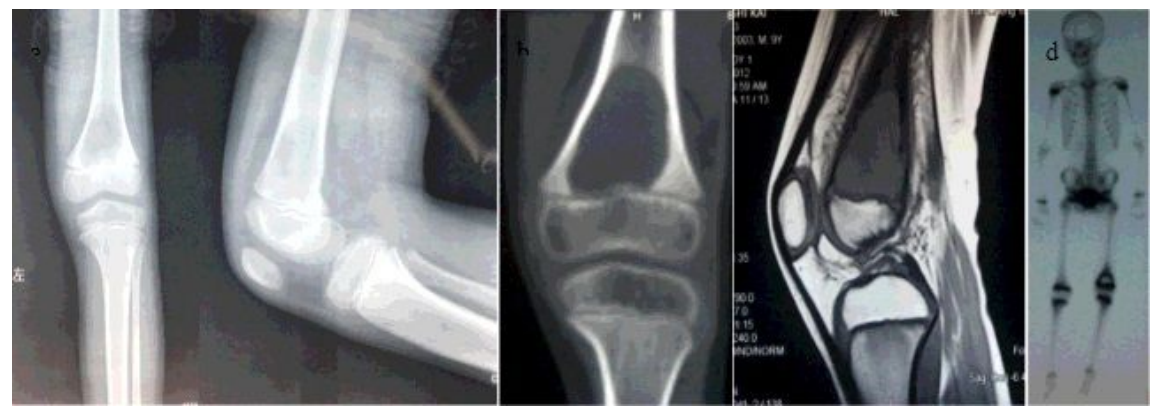

\section{Figure 3}

The procedure for the resection and reconstruction of the osteosarcoma with ransepiphyseal osteotomy by dependent design of 3D-printed guide plate and prosthesis. (a-b) Resection of the osteosarcoma with the assistance of 3D-guide plate. (c-e) Reconstruction of the bone defect with dependent design of 3Dprinted prosthesis. (f) Photograph of the tumor specimen.
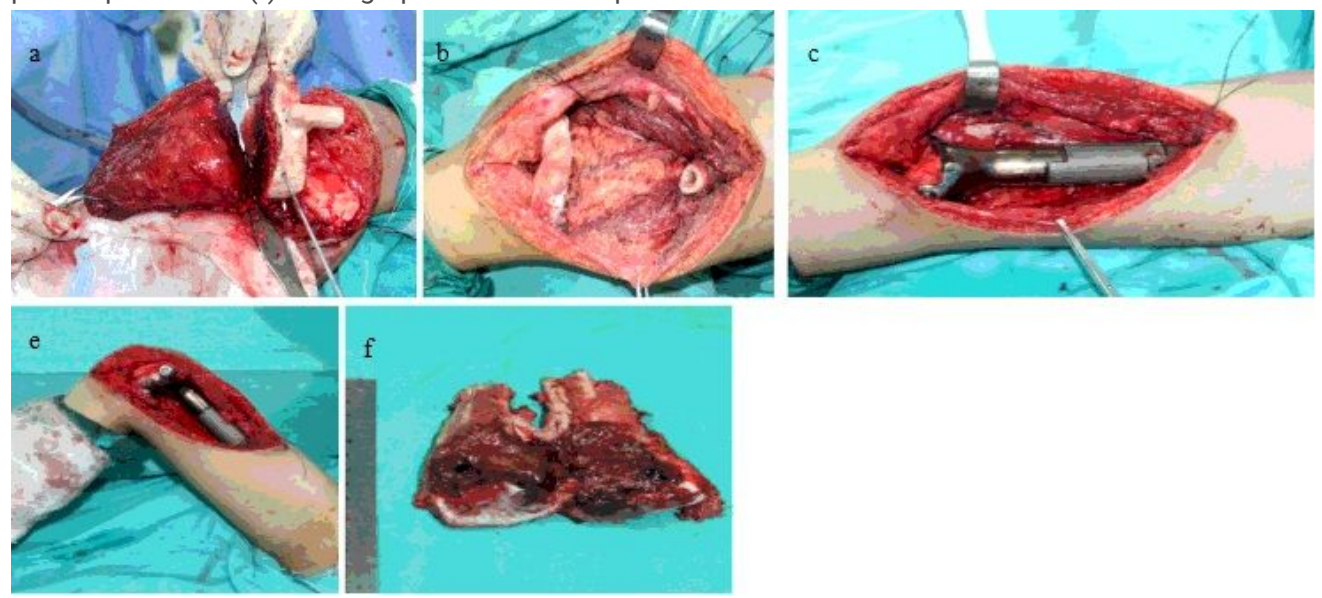

\section{Figure 4}

The outcome of the patient with seven years follow-up. (a-c) The X-ray for the knee joint and prosthesis with the follow-up of one year, four years and seven years. (d-f) Photographs about the length and function of the patient at the follow-up of seven years 